\title{
Smooth Output Reconstruction for Linear Systems with Quantized Measurements
}

\section{$\operatorname{AUTHOR}(\mathrm{S})$ :}

Zhu, Hongzhong; Sugie, Toshiharu; Fujimoto, Hiroshi

\section{CITATION:}

Zhu, Hongzhong ... [et al]. Smooth Output Reconstruction for Linear Systems with Quantized Measurements. Asian Journal of Control 2015, 17(3): 1039-1049

\section{ISSUE DATE:}

2015-05

URL:

http://hdl.handle.net/2433/203540

\section{RIGHT:}

This is the accepted version of the following article: Zhu, H., Sugie, T., and Fujimoto, H. (2015), Smooth Output Reconstruction for Linear Systems with Quantized Measurements. Asian Journal of Control, 17, 1039-1049, which has been published in final form at http://dx.doi.org/10.1002/asjc.950. This article may be used for non-commercial purposes in accordance with Wiley Terms and Conditions for Self-Archiving.; The full-text file will be made open to the public on 31 July 2015 in accordance with publisher's 'Terms and Conditions for Self-Archiving'.; This is not the

published version. Please cite only the published version.; この論文は出版社版でありません。引用の際には出版社版 をご確認ご利用ください。 


\title{
Smooth Output Reconstruction for Linear Systems with Quantized Measurements
}

\author{
Hongzhong Zhu, Toshiharu Sugie, Hiroshi Fujimoto
}

\begin{abstract}
This paper presents a novel approach to reconstruct the output of linear systems in the case where the measured output is uniformly quantized. By fitting the quantized measurements with polynomials in a moving horizon manner, a smooth signal is reconstructed by solving a convex optimization problem with $\ell_{1}$-norm regularization. The quantization feature and the system models are taken into account in the optimization. A numerical example is given to show the excellent reconstruction performance of the proposed method. In addition, the proposed method is implemented in a high-precision linear stage through DSP, and its effectiveness is verified through experiments using a real positioning system.
\end{abstract}

Key Words: quantization; polynomial fitting approach; convex optimization; state observer

\section{Introduction}

Quantization in I/O signals is an inherent feature in many control systems, including digital systems, networked systems and low resolution sensor/actuator systems. In some cases, the quantization error is relatively small compared to system noise and the desired control accuracy. Nevertheless, this is not always the case in various systems, such as computer storage systems, NC machine tools, industrial robots, and ultra-precision positioning systems, where the required accuracy is nano order. In these systems, the quantization error caused by low-resolution sensors could significantly degrade the control performance and may cause limit cycle oscillations [1]. In addition, in networked control systems with limited communication capacity, sensor measurement is quantized before transmission. In such cases, the quantization error may

Manuscript received January 25, 2013; revised June 10, 2013; accepted December 21, 2013.

H. Zhu and H. Fujimoto are with the Department of Electrical Engineering, The University of Tokyo, Kashiwa, Chiba 2778561 Japan (e-mails:zhu@hflab.k.u-tokyo.ac.jp, fujimoto@k.utokyo.ac.jp).

T. Sugie is with the Department of System Science, Kyoto University, Uji, Kyoto 611-0011 Japan (e-mail: sugie@i.kyotou.ac.jp). not be ignored $[2,3,4]$. Therefore, it is necessary to consider the signal quantization effect explicitly $[5,6$, $7,8,9,10]$.

In order to cope with the quantization effects, various methods have been proposed in the literature to reconstruct the real system output. One strategy is to utilize the system model information. For instance, some observer-based methods have been proposed to estimate the system state, and a reconstruction output can be obtained by utilizing the estimated state and the model information [11, 12]. Hirata et al. proposed a method to estimate the quantization error directly via the least square method in the presence of a constant disturbance [13]. Nevertheless, since these methods strongly depend on the system models, the accuracy of the reconstructed output could be degraded drastically when the models are not precise. Also, it is desirable to handle not only the constant disturbances but also the other types of disturbances.

There is another line of research on reconstructing the real system output from the noise-corrupted output by curve fitting methods. For instance, polynomial filtering approaches have been proposed to recover non-uniformly sampled signals [14] and position signals obtained from incremental encoders [15]. The methods can work well if the output signal can 
be locally approximated by low-degree polynomials. Nevertheless, if this is not the case, higher-degree polynomials are required. Hence, it would be difficult to adopt these methods. Moreover, how to determine the degree of the polynomials was not clear in the literature.

Based on the above observation, Zhu et al. proposed a method to estimate the velocity of motion systems based on low-resolution encoders [16]. The method combines a curve fitting approach with the observer-based ones. It is shown that the method outperforms the conventional Kalman filter approach. In fact, it achieves smooth velocity reconstruction with high precision even if the encoder resolution is extremely low. Furthermore, its effectiveness has been demonstrated through DC motor positioning servo experiments. Nevertheless, it considers the velocity estimation of second order mechanical systems only, and the disturbance was assumed to be zero mean, which may be too restrictive in many practical systems. Also, the experiments were conducted using MATLAB and RTW toolbox package, hence it is not clear if it works in field applications where the algorithm should be implemented in, e.g., DSP.

The purpose of this paper is to extend the method proposed in [16] to output reconstruction of general linear systems while overcoming the above drawbacks. More precisely, the contribution of this paper is as follows. (a) A new method for general linear systems is given to reconstruct the smooth output from the quantized measurement in the presence of (nonzero mean) system disturbances based on [16]. The method to reconstruct the output uses moving horizon polynomial curve fitting, where $\ell_{1}$-norm regularization is adopted to determine the appropriate polynomial curve and the information of the system models is exploited. (b) The proposed method is implemented in a real high-precision linear stage through DSP, and its effectiveness is verified though the experiments of positioning control.

This paper is organized as follows. In Section II, the system model is introduced and the problem setting is described. Section III presents the polynomial fitting approach based on convex optimization that consists of the $\ell_{1}$-norm regularization method and some constraint conditions. A numerical example is given in Section IV to illustrate the advantages of the proposed approach. In Section V, the proposed algorithm is implemented in DSP and its effectiveness is demonstrated through experiments using a high-precision positioning stage. The conclusions are summarized in Section VI.

\section{System description}

Consider the linear time-invariant SISO system with quantized output given by:

$$
\begin{aligned}
& \boldsymbol{x}[k+1]=\boldsymbol{A} \boldsymbol{x}[k]+\boldsymbol{B} u[k]+\boldsymbol{F} w[k], \\
& y[k]=\boldsymbol{C} \boldsymbol{x}[k], \\
& y_{v}[k]=y[k]+v[k], \\
& y_{q}[k]=\boldsymbol{Q}\left(y_{v}[k]\right),
\end{aligned}
$$

where $\boldsymbol{x} \in \mathbb{R}^{n}, y \in \mathbb{R}, y_{v} \in \mathbb{R}$ and $y_{q} \in \mathbb{R}$ are the system state, the system output, the corrupted output and the quantized output, respectively. $v \in \mathbb{R}$ is the measurement noise, and $w \in \mathbb{R}$ is the unknown input disturbance. $\boldsymbol{A}, \boldsymbol{B}, \boldsymbol{F}, \boldsymbol{C}$ are constant system matrices of appropriate dimensions. $\boldsymbol{Q}(\cdot)$ denotes the quantization. $v$ is assumed to be zero-mean and bounded as $\|v\|_{\infty} \leq \delta$ with known $\delta$.

The disturbance $w$ is assumed to be expressed by:

$$
\begin{aligned}
& \boldsymbol{\zeta}[k+1]=\boldsymbol{\Gamma} \boldsymbol{\zeta}[k], \\
& w[k]=\boldsymbol{H} \boldsymbol{\zeta}[k],
\end{aligned}
$$

where $\zeta$ denotes the disturbance state and where $\boldsymbol{\Gamma}$ and $\boldsymbol{H}$ are real matrices of suitable dimensions. Note that such $w$ may include step and/or periodic disturbances, which can be seen in many practical systems. Defining the augmented state $\boldsymbol{x}_{e}$ as $\boldsymbol{x}_{e}:=$ $\left[\begin{array}{ll}\boldsymbol{x}^{T} & \boldsymbol{\zeta}^{T}\end{array}\right]^{T}$, the augmented system of (1), (2), (3), (4) can be expressed by:

$$
\begin{aligned}
& \boldsymbol{x}_{e}[k+1]=\boldsymbol{A}_{e} \boldsymbol{x}_{e}[k]+\boldsymbol{B}_{e} u[k], \\
& y_{v}[k]=\boldsymbol{C}_{e} \boldsymbol{x}_{e}[k]+v[k], \\
& y_{q}[k]=\boldsymbol{Q}\left(y_{v}[k]\right),
\end{aligned}
$$

where

$$
\begin{aligned}
\boldsymbol{A}_{e} & =\left[\begin{array}{cc}
\boldsymbol{A} & \boldsymbol{F} \boldsymbol{H} \\
\mathbf{0} & \boldsymbol{\Gamma}
\end{array}\right], \boldsymbol{B}_{e}=\left[\begin{array}{c}
\boldsymbol{B} \\
0
\end{array}\right], \\
\boldsymbol{C}_{e} & =\left[\begin{array}{ll}
\boldsymbol{C} & 0
\end{array}\right] .
\end{aligned}
$$

In addition, the function $Q(\cdot)$ in (4) is assumed as the uniform quantization defined by:

$$
\boldsymbol{Q}\left(y_{v}\right)=i \cdot \Delta, \quad y_{v} \in((i-0.5) \Delta,(i+0.5) \Delta] \text { (10) }
$$

where $i \in \mathbb{Z}, \Delta>0$ denotes the quantization step. The relationship between $y_{v}$ and $y_{q}$ is shown in Figure 1. The quantizer (10) could represent low-resolution optical encoders or analog-to-digital converters in real systems, where $\Delta$ is referred to as the resolution.

Due to the measurement noise $v$, the difference between the system output $y$ and the measured quantized output $y_{q}$, denoted by $\xi:=y-y_{q}$, is bounded by:

$$
|\xi| \leq \frac{\Delta}{2}+\delta
$$




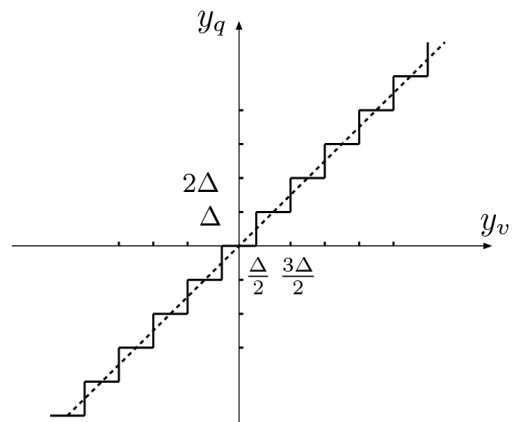

Fig. 1. Quantization characteristic. $\Delta$ is the quantization step.

\section{Algorithm for output reconstruction}

In this section, an output reconstruction approach is proposed. First, the quantized measurements are locally fitted by a polynomial based on $\ell_{1}$-norm regularization method. Then, the moving horizon manner is applied to achieve the real-time output reconstruction. Finally, some constraint conditions taking advantage of the quantization feature and observer techniques are adopted to achieve smooth reconstruction signals.

\subsection{Polynomial fitting formulation}

A polynomial for fitting $p+1$ quantized measurements $\left(\left\{y_{q}[k-i]\right\}_{i=0,1, \cdots, p}\right)$ is considered. Here, $k$ denotes the current time instant. In order to do so, first, choose the time interval $\left[\begin{array}{ll}a & b\end{array}\right]$ in advance and introduce the virtual time indices $\left\{\tau_{i}\right\}_{i=0,1, \cdots, p}$, which are defined by

$$
\tau_{0}=a, \tau_{1}=a+h, \cdots, \tau_{i}=a+i h, \cdots, \tau_{p}=b,
$$

where $h=(b-a) / p$, to equally divide $\left[\begin{array}{ll}a & b\end{array}\right]$. Then, the data $\left(\tau_{i}, y_{q}[k-p+i]\right)_{i=0,1, \cdots, p}$ are fitted in Euclidean space with the polynomial:

$$
g_{k}(t)=\alpha_{0}+\alpha_{1} \tau+\alpha_{2} \tau^{2}+\cdots+\alpha_{m} \tau^{m},
$$

where $\tau \in\left[\begin{array}{ll}a & b\end{array}\right], \alpha_{0}, \alpha_{1}, \cdots \alpha_{m}$ are the polynomial coefficients and $m$ is the degree of the polynomial. Without loss of generality, $m$ is assumed to be $m \leq$ $p+1$. The fitting problem is formulated by:

$$
\min _{\boldsymbol{\alpha}}:\left\|\left[\begin{array}{c}
g_{k}\left(\tau_{0}\right)-y_{q}[k-p] \\
\vdots \\
g_{k}\left(\tau_{i}\right)-y_{q}[k-p+i] \\
\vdots \\
g_{k}\left(\tau_{p}\right)-y_{q}[k]
\end{array}\right]\right\|_{2}^{2}+\eta\|\boldsymbol{\alpha}\|_{1},
$$

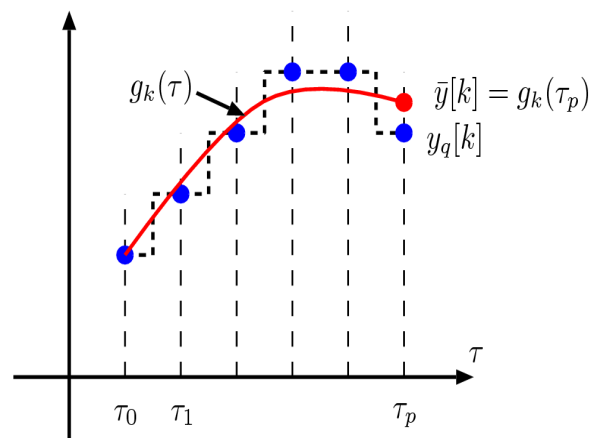

Fig. 2. Fitting strategy. The latest $p+1$ quantized measurements are locally fitted by a polynomial $g_{k}(\tau) . g_{k}\left(\tau_{p}\right)$ is regarded as the reconstruction output at time instant $k$.

with variable $\boldsymbol{\alpha}:=\left[\begin{array}{llll}\alpha_{0} & \alpha_{1} & \cdots & \alpha_{m}\end{array}\right]^{T}$. Here, $\eta$ is the weighting factor. This is an $\ell_{1}$-norm regularization problem and can be expressed by:

$$
\min _{\boldsymbol{\alpha}}:\|\boldsymbol{T} \boldsymbol{\alpha}-\boldsymbol{\beta}\|_{2}^{2}+\eta\|\boldsymbol{\alpha}\|_{1},
$$

where

$\boldsymbol{T}=\left[\begin{array}{cccc}1 & \tau_{0} & \cdots & \tau_{0}^{m} \\ \vdots & \vdots & \ddots & \vdots \\ 1 & \tau_{i} & \cdots & \tau_{i}^{m} \\ \vdots & \vdots & \ddots & \vdots \\ 1 & \tau_{p} & \cdots & \tau_{p}^{m}\end{array}\right], \boldsymbol{\beta}=\left[\begin{array}{c}y_{q}[k-p] \\ \vdots \\ y_{q}[k-p+i] \\ \vdots \\ y_{q}[k]\end{array}\right]$.

Figure 2 demonstrates the fitting strategy. Note that (14) becomes a normal least-square polynomial fitting problem if $\eta=0$. Regularization with $\ell_{1}$-norm can be used to find a sparse solution of $\boldsymbol{\alpha}$ [17]. In this way, the unnecessary terms of the polynomial (12) can be removed automatically if a high-degree polynomial is used to fit a simple signal. In practical situations, $m$ can be relatively large so that simple signals, as well as complex signals, could be properly fitted.

In general, the time indices $\left\{\tau_{i}\right\}_{i=0,1, \cdots, p}$ are chosen as the real time instants. In such a case, however, the Vandermonde matrix $\boldsymbol{T}$ is time-varying and would become ill-condition, so the fitting accuracy might be degraded. In order to cope with the problem, a fixed symmetric interval with respect to the origin is chosen to make $\boldsymbol{T}$ well-condition [18]. Without loss of generality, $\left[\begin{array}{ll}a & b\end{array}\right]$ is chosen as $\left[\begin{array}{ll}-1 & 1\end{array}\right]$ in this study.

\subsection{Moving horizon manner}

Once the convex optimization problem (14) is solved, the polynomial (12) is determined. Hence, the quantity $\bar{y}[k]$ is given by:

$$
\bar{y}[k]:=g_{k}\left(\tau_{p}\right)=\boldsymbol{\tau}_{p}^{T} \boldsymbol{\alpha},
$$




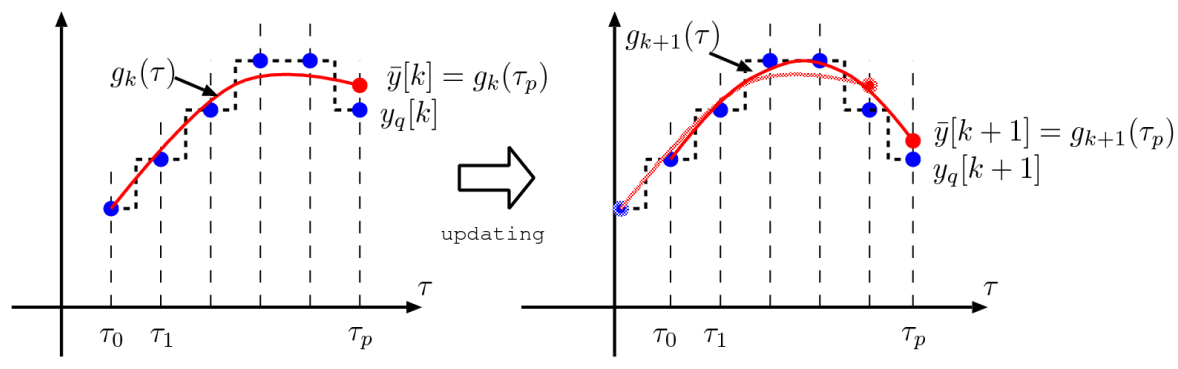

Fig. 3. Updating strategy. A new polynomial is calculated when the time instant is updated.

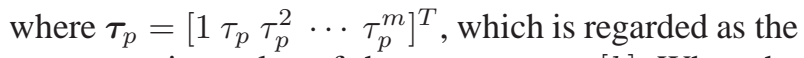
reconstruction value of the true output $y[k]$. When the time instant $k$ is updated, say, from $k$ to $k+1$, a new quantized measurement $y_{q}[k+1]$ is sampled. Then, a new polynomial $g_{k+1}(\tau)$ can be determined by fitting the new data $\left\{y_{q}[k-p+1], y_{q}[k-p+2], \cdots, y_{q}[k+\right.$ $1]\}$. The updated quantity $\bar{y}[k+1]$ is calculated by:

$$
\bar{y}[k+1]=g_{k+1}\left(\tau_{p}\right) .
$$

Figure 3 shows the updating strategy. As shown above, $\bar{y}[k]$ is determined from the data $\left\{y_{q}[k-p], y_{q}[k-p+\right.$ $\left.2], \cdots, y_{q}[k]\right\}$ in the moving horizon manner. In the case of $k \leq p, y_{q}[k-i](i=k, k+1, \cdots, p)$ is set as $y_{q}[k-i]:=0$.

\subsection{Constraint conditions}

In order to improve the reconstruction accuracy, some constraint conditions are added in the optimization problem (14) by taking advantage of the model information.

First, since the discrepancy between the system output $y[k]$ and the corresponding quantized output $y_{q}[k]$ is bounded by (11), the quantity $\bar{y}[k]$ should be bounded by:

$$
\left|\bar{y}[k]-y_{q}[k]\right| \leq \frac{\Delta}{2}+\delta .
$$

According to (12) and (15), this condition can be expressed by:

$$
\left|\boldsymbol{\tau}_{p}^{T} \boldsymbol{\alpha}-y_{q}[k]\right| \leq \frac{\Delta}{2}+\delta .
$$

Note that the left hand side of (17) is convex with respect to $\alpha$.

Second, the fitting curve $g_{k}(t)$ (at time instant $k$ ) should take $\bar{y}[k-1]$ into account as well as the quantized output sequence $y_{q}[i](i=k, k-1, \cdots, k-$ $p$ ). In addition, the system information (including the disturbance model) should be taken into account. Let $\hat{y}[k]$ be the output of the observer for the augmented system (7) (8). Namely,

$$
\begin{aligned}
& \hat{\boldsymbol{x}}[k+1]=\boldsymbol{A}_{e} \hat{\boldsymbol{x}}[k]+\boldsymbol{B}_{e} u[k]+\boldsymbol{L}_{e}(\bar{y}[k]-\hat{y}[k]), \\
& \hat{y}[k]=\boldsymbol{C}_{e} \hat{\boldsymbol{x}}[k],
\end{aligned}
$$

where $\boldsymbol{L}_{e} \in \mathbb{R}^{(n+1) \times 1}$ is the observer gain to stabilize $\boldsymbol{A}_{e}-\boldsymbol{L}_{e} \boldsymbol{C}_{e}$. Then, the following two constraints are added.

$$
\begin{aligned}
g_{k}\left(\tau_{p-1}\right) & =\hat{y}[k-1], \\
\dot{g}_{k}\left(\tau_{p-1}\right) & =\frac{1}{h}(\hat{y}[k]-\hat{y}[k-1])
\end{aligned}
$$

where $\dot{g}_{k}(\tau)$ is the first derivative of the polynomial (12), which is given by

$$
\dot{g}_{k}(\tau)=\alpha_{1}+2 \alpha_{2} \tau+\cdots+m \alpha_{m} \tau^{m-1} .
$$

Equation (20) indicates that $\hat{y}[k-1]$ and $\bar{y}[k]$ belong to $g_{k}(\tau)$ and that Equation (21) is a slope condition for obtaining smooth reconstruction. Note that the left hand sides of (20) and (21) are also affine to $\boldsymbol{\alpha}$. For the implementation of real-time calculation, $\hat{y}[k]$ is computed via:

$$
\begin{array}{r}
\hat{y}[k]=\boldsymbol{C}_{e}\left[\boldsymbol{A}_{e} \hat{\boldsymbol{x}}[k-1]+\boldsymbol{B}_{e} u[k-1]+\right. \\
\left.\boldsymbol{L}_{e}(\bar{y}[k-1]-\hat{y}[k-1])\right] .
\end{array}
$$

\subsection{System output reconstruction}

The quantized output reconstruction algorithm is summarized as follows.

Algorithm: Output reconstruction

1. Choose proper values for $p, m$, $\eta$; set initial value for $\boldsymbol{\beta}$ as $\boldsymbol{\beta}=0$, assign the values for $\Delta, \delta$;

\section{2. repeat}




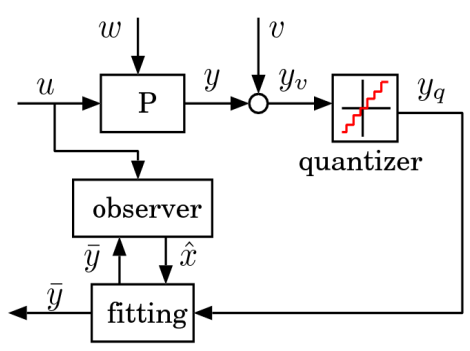

Fig. 4. Block diagram of the proposed approach, $\bar{y}$ is regarded as the reconstruction of $y$.

(a) Sample the position measurement $y_{q}[k]$, update the vector $\boldsymbol{\beta}$;

(b) solve the problem

$$
\min _{\boldsymbol{\alpha}}: \quad\|\boldsymbol{T} \boldsymbol{\alpha}-\boldsymbol{\beta}\|_{2}^{2}+\eta\|\boldsymbol{\alpha}\|_{1}
$$

subject to : (17), (20), (21)

to obtain $\boldsymbol{\alpha}$

(c) calculate $\bar{y}[k], \hat{\boldsymbol{x}}[k]$ by (15), (18) and (19);

(d) $\operatorname{set} k \leftarrow k+1$.

The optimization problem (24) is a convex optimization problem and can be solved efficiently. Note that the three constraint conditions are independent of each other so the problem is feasible if the polynomial (12) has a degree not less than $2(m \geq 2)$. The block diagram of the proposed approach is shown in Figure 4.

\subsection{Discussions}

From (11) and (16), it is immediate to have

$$
|\bar{y}[k]-y[k]| \leq \Delta+2 \delta, \quad \forall k .
$$

Hence, the construction error is guaranteed to be bounded, and the control system is BIBO stable if the unquantized system is stable. Unfortunately, since the quantization error behaves as highly colored noise so that the asymptotic convergence cannot be obtained by polynomial fitting approach, it is difficult to give a tighter error bound analytically, which is similar to the existing results [19]. Nevertheless, it is intuitively clear that the reconstructed output $\bar{y}[k]$ is much closer to $y[k]$ compared to $y_{q}[k]$, because $\bar{y}[k]$ takes account of both the smooth maneuver of $y[k]$ (due to the system dynamics) and the constraints given by the augmented system model. In fact, according to our extensive simulation/experimental study, the proposed method yields surprisingly precise output estimation in almost all cases. Hence, it would be beneficial to illustrate its performance through some numerical examples.

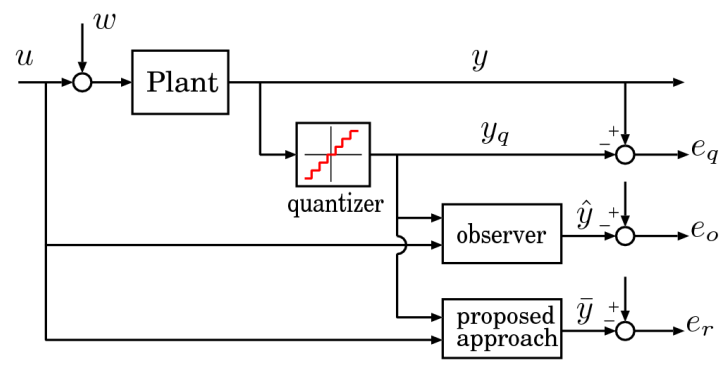

Fig. 5. Block diagram of the quantized system for simulation.

\section{Illustrative examples}

In this section, one numerical example is given to illustrate the effectiveness of the proposed method. Consider the system given by:

$$
\begin{aligned}
& \boldsymbol{x}[k+1]=\boldsymbol{A} \boldsymbol{x}[k]+\boldsymbol{B}(u[k]+w[k]), \boldsymbol{x}[0]=\mathbf{0}, \\
& y[k]=\boldsymbol{C} \boldsymbol{x}[k],
\end{aligned}
$$

where

$$
\begin{gathered}
\boldsymbol{A}=\left[\begin{array}{rrr}
1.00000 & 0.00001 & 0 \\
0 & 1.00000 & 0.00001 \\
0 & -0.00020 & 0.99930
\end{array}\right], \\
\boldsymbol{B}=\left[\begin{array}{r}
0 \\
0 \\
0.00030
\end{array}\right], \quad \boldsymbol{C}=\left[\begin{array}{lll}
1 & 0 & 0
\end{array}\right],
\end{gathered}
$$

and $u=30 \sin \frac{2 \pi k}{10^{5}}, k \leq 10^{5}$. The evaluation for the proposed approach is shown in Figure 5. In the proposed method, the order of the polynomial in (12) is set as $m=4$, the number of quantized output used for fitting is $p+1=50$, and the weight factor $\eta$ is $\eta=$ $1 \times 10^{-3}$. The observer gain $\boldsymbol{L}_{e}$ in (18) (19) is properly given as $[0.00070 .02140 .00150 .3599]^{T}$.

We compare the accuracy of three output estimates:

(a) Quantized output $y_{q}$;

(b) An observer-based output estimate $\hat{y}$;

(c) The reconstructed output $\bar{y}$ by the proposed method.

In the above (b), $\hat{y}[k]$ is calculated from $u[k]$ and $y_{q}[k]$ using the same observer (18) (19) (except that $\bar{y}[k]$ is replaced by $\left.y_{q}[k]\right)$. The errors used for evaluation are defined by:

$$
\begin{aligned}
& e_{q}:=y_{q}-y, \\
& e_{o}:=\hat{y}-y, \\
& e_{r}:=\bar{y}-y,
\end{aligned}
$$




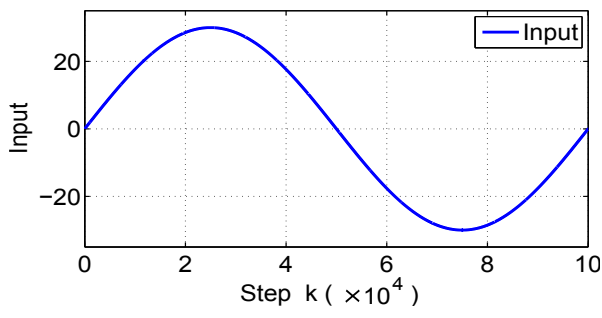

Fig. 6. Input $u$ for the system shown in Figure 5.

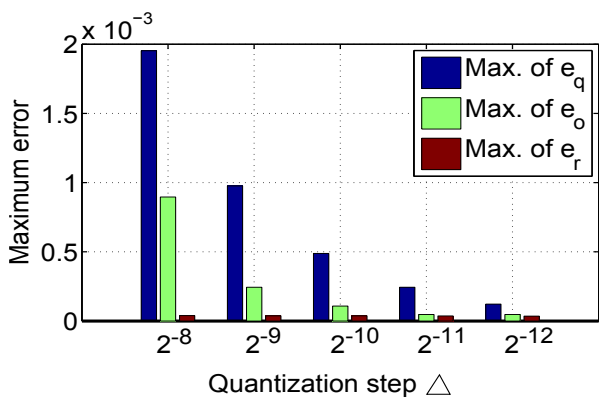

Fig. 7. Comparison of the maximum errors.

where $e_{q}$ denotes the quantization error, $e_{o}$ denotes the estimation error of the observer, and $e_{r}$ denotes the reconstruction error of the proposed approach.

First, the disturbance is set as $w[k]=$ $0, \quad \forall \quad k$, and the maximum errors, namely $\max _{k}\left|e_{q}[k]\right|, \quad \max _{k}\left|e_{o}[k]\right|$ and $\quad \max _{k}\left|e_{r}[k]\right|$, are calculated for various quantization steps $\Delta \in\left\{2^{-8}, 2^{-9}, \cdots, 2^{-12}\right\}$. The comparison is shown in Figure 7. It shows that the reconstruction error of the proposed approach is much smaller than $e_{q}$ and $e_{o}$. Also, the reconstruction accuracy is almost insensitive to the quantization step $\Delta$. Figure 8 shows an example of time trajectories of $e_{q}, e_{o}$, and $e_{r}$ explicitly in the case of $\Delta=2^{-8}$. The reconstruction error $e_{r}$ is very small compared to the quantization error $e_{q}$ and the estimation error $e_{o}$. Figure 7 and Figure 8 illustrate the effectiveness of the proposed approach.

Then, the dynamic response of the proposed method in the presence of disturbance $w$ is examined. A step signal $w[k]=5, k \geq 2 \times 10^{4}$ is introduced into the system at the time instant $k=2 \times 10^{4}$. The numerical results are shown in Figure 9. Figure 9(a) shows the comparison of the added disturbance (solid line) and the estimated disturbance (dashed line). It is demonstrated that the disturbance also can be estimated excellently by the proposed approach after the convergence. This is important because we can suppress their effects using the accurate estimation of the disturbances. Figure 9(b) shows the comparison of the quantization error $e_{q}$ and

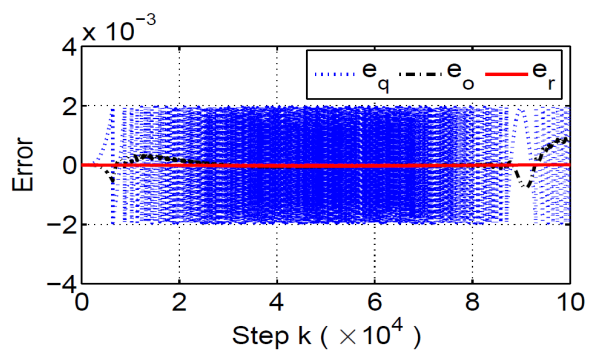

Fig. 8. Simulation result when quantization step is set as $\Delta=2^{-8}$. The dotted line shows the quantization error $e_{q}$, the dot-dashed line shows the estimated error $e_{o}$ by the observer, and the solid line shows the reconstruction error $e_{r}$ of the proposed approach.

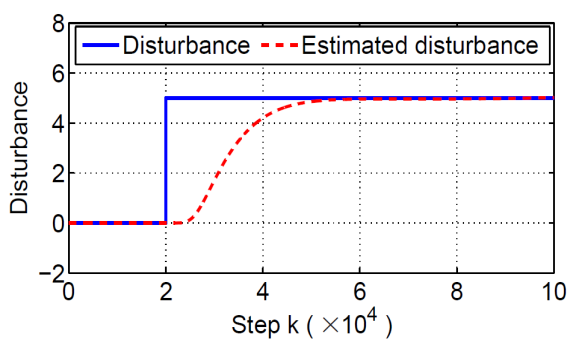

(a) Comparison of disturbance and estimated disturbance.

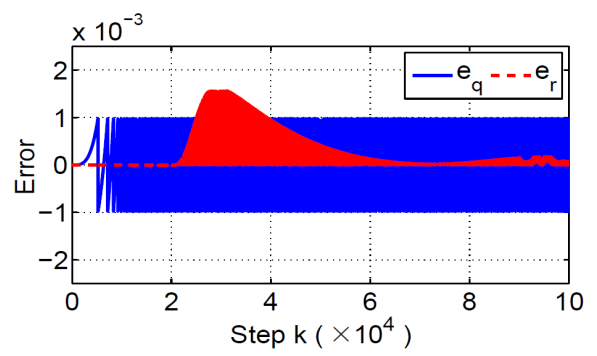

(b) Reconstruction error when disturbance is added.

Fig. 9. Simulation results when step disturbance is added at $k=2 \times$ $10^{4}$.

the reconstruction error $e_{r}$. Although $e_{r}$ could be larger than the quantization error during the transient process, it converges gradually and becomes much smaller than the quantization error. This implies that the proposed method is effective against disturbances with non-zero mean. 


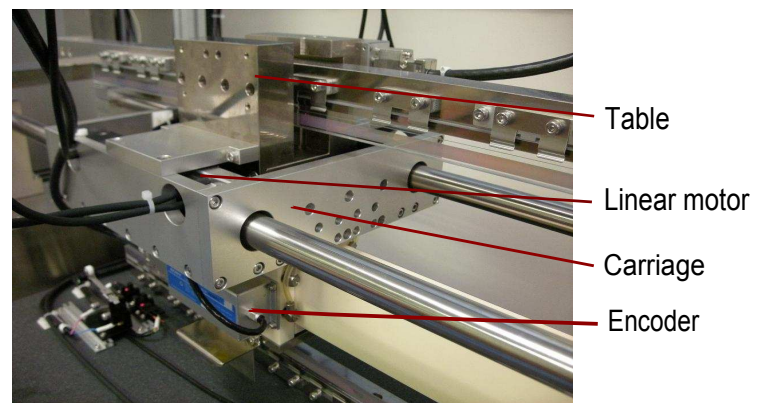

Fig. 10. Experimental setup.

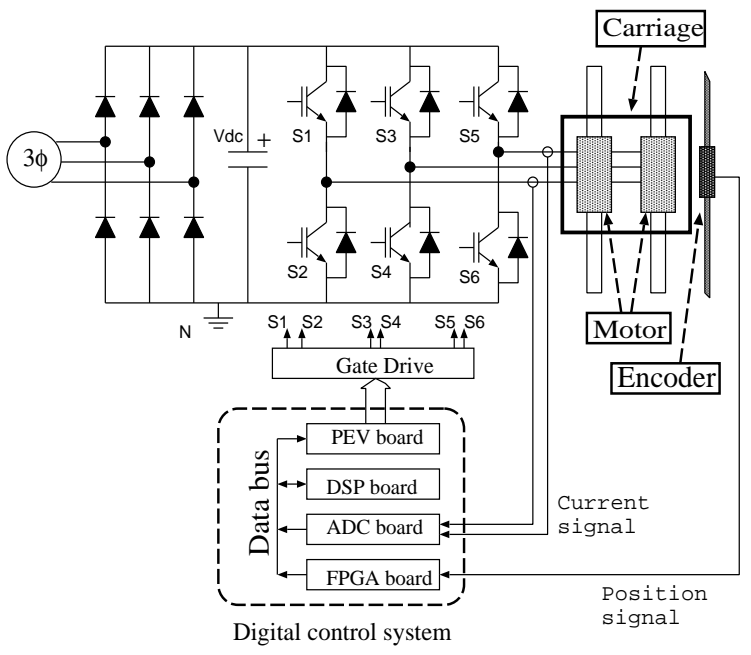

Fig. 11. Configuration of experimental system.

\section{Experimental validation}

In this section, the effectiveness of the proposed method will be validated through experiments using a high precision stage.

\subsection{Description of the experiment system}

The high precision stage is shown in Figure 10. The stage is a simplified model of the scanner in exposure systems used for the fabrication of integrated circuits, which is required to achieve extremely precise motion control. The configuration of the experimental system is shown in Figure 11. Two linear motors located at both sides of the carriage are applied to drive the stage. An air guide system is introduced to reduce the friction between the stator and the slider of the motors. DSP(TMS320C6713, 225MHz) is used as the processor to implement the controllers and the proposed fitting

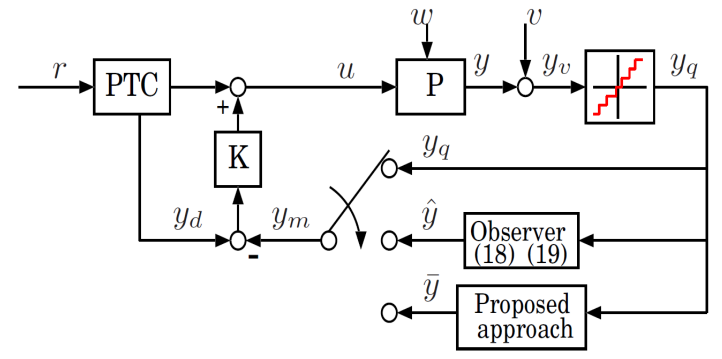

Fig. 12. Block diagram of the control system.

approach. The nominal model is expressed as:

$$
P(s)=\frac{26.5}{s(14.7 s+24)} \text {. }
$$

\subsection{Control system}

A two-degree-of-freedom controller is exploited to control the stage, and the block diagram of the control system is shown in Figure 12. The feed forward controller, designed by the perfect tracking control (PTC) method [20], is the stable inverse system of the nominal plant, so perfect tracking at every sampling instant can be guaranteed. The feedback controller is designed as the PID compensator given by:

$$
K=k_{p}+k_{d} \frac{s}{0.004 s+1}+k_{i} \frac{1}{s}
$$

where $k_{p}=2065.6, k_{i}=34624, k_{d}=43.45$ and the bandwidth of the close-loop system is $10 \mathrm{~Hz}$. In the figure, $y_{d}$ denotes the desired position trajectory and $y_{m}$ denotes the measurement position used for feedback. The control system is discretized with the sampling period $T_{s}=5 \mathrm{~ms}$. Denote $\boldsymbol{x}$ as $\boldsymbol{x}:=\left[\begin{array}{ll}y & \dot{y}\end{array}\right]^{T}$, where $y, \dot{y}$ are the position and the velocity of the stage, respectively. The discrete-time state space expression of (31) using the zero-order-hold method is given by:

$$
\begin{aligned}
& \boldsymbol{x}[k+1]=\boldsymbol{A} \boldsymbol{x}[k]+\boldsymbol{B}(u[k]+w[k]), \\
& y[k]=\boldsymbol{C} x[k],
\end{aligned}
$$

where

$$
\begin{aligned}
& \boldsymbol{A}=\left[\begin{array}{rr}
1 & 0.0050 \\
0 & -0.9919
\end{array}\right], \boldsymbol{B}=\left[\begin{array}{l}
2.247 e^{-5} \\
8.977 e^{-3}
\end{array}\right], \\
& \boldsymbol{C}=\left[\begin{array}{ll}
1 & 0
\end{array}\right],
\end{aligned}
$$

and $w$ is the input disturbance. According to the real situation, $w$ is treated as a step disturbance. Therefore, $\boldsymbol{\Gamma}$ and $\boldsymbol{H}$ in the disturbance state equation (5) (6) are chosen as $\boldsymbol{\Gamma}=1$ and $\boldsymbol{H}=1$. 
Although a linear encoder with a resolution of $1 \mathrm{~nm}$ is available for the position measurement in this system, we do not use it for control purposes. Instead, a software quantizer is introduced, whose resolution (i.e., quantization step) is $\Delta=20 \mu \mathrm{m}$, in order to evaluate the effectiveness of the proposed method. The quantized output $y_{q}$ (with a resolution of $20 \mu \mathrm{m}$ ) is supposed to be used for control. The high-resolution encoder is used only for monitoring the performance precisely. In this way, the measurements from the linear encoder are regarded as the actual output $y$, and the signal from the software quantizer is treated as the quantized output $y_{q}$.

\subsection{Implementation of the proposed method}

According to the algorithm given in Section 3.4, we implement the proposed method to yield $\bar{y}$ based on $u$ and $y_{q}$. The number of data used for polynomial fitting is set as $p+1=15$, and the degree of the polynomial is set as $m=3$. The weight factor $\eta$ is properly chosen as $\eta=2 \times 10^{-3}$. $\delta$ is set as $2 \times 10^{-9}$ by trial and error. The C-code of solving the convex optimization problem (24) is generated by CVXGEN [21]. The gain $\boldsymbol{L}_{e}$ of the state estimator is determined by placing the poles of the observer at $[0.5335,0.6242,0.7304]$, which results in the observer bandwidth being $20 \mathrm{~Hz}$. The reconstruction algorithm is implemented in the DSP. By this setting, the average computational time of solving the convex optimization problem (24) is about $3 \mathrm{~ms}$, which shows the problem can be solved safely during the sampling period $T=5 \mathrm{~ms}$.

\subsection{Experimental evaluation}

We perform experiments for the following three cases.

Case I $y_{q}$ is used directly as the measurement $y_{m}$.

Case II $\hat{y}$ is used for the measurement $y_{m}$, where $\hat{y}$ is the output of the observer given by (18) and (19) with the input $y_{q}$ instead of $\bar{y}$.

Case III $\bar{y}$ is used as the measurement $y_{m}$.

For comparison, the position tracking error also is defined:

$$
e_{t}:=y_{d}-y \text {. }
$$

In order to examine the transient and the settling performance of the stage, the trajectory reference, including acceleration, uniform motion, deceleration, and settling motion, is given, as shown in Figure 13. The experimental results for Cases I, II, and III are shown

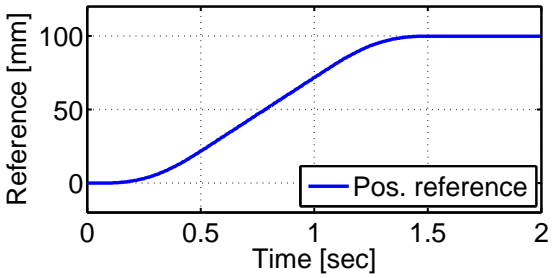

Fig. 13. Trajectory reference.

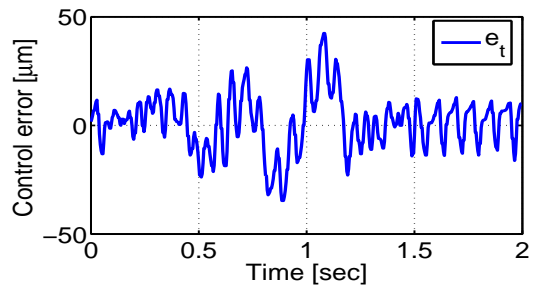

(a)

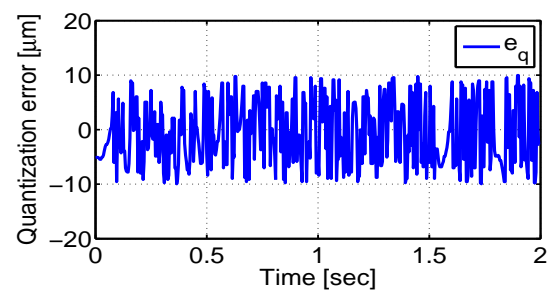

(b)

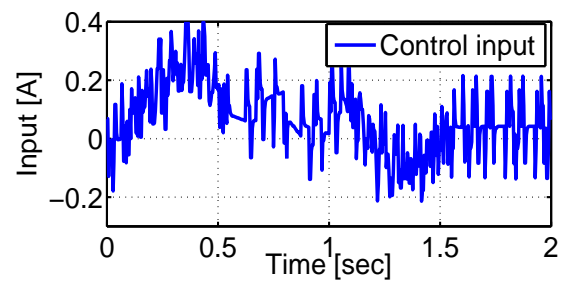

(c)

Fig. 14. Experimental results of Case I. (a) shows the position tracking error. (b) shows the quantization error, and (c) shows the control input.

in Figure 14, Figure 15, and Figure 16, respectively. In the figures, (a)s show the control errors $e_{t}$. (b)s in Figure 15 and Figure 16 show the comparison of $e_{q}$ and $e_{o} / e_{r}$. Finally, (c)s show the control inputs. It is 
observed that the high-order vibration is excited not only at the transient process, but also in the settling motion area in Case I. In Case II, the estimated error $e_{o}$ can be larger than the quantization step. Hence, it may further degrade the control performance, as shown in Figure 15(a) (compared to Figure 14(a)). Figure 16(b) shows that the reconstruction error is much smaller than the quantization error, which implies that the accuracy of the feedback signal is improved. This is also reflected in Figure 16(a), where the high-order vibration excited by the quantization error is suppressed by the proposed method. The estimated disturbance is shown in Figure 16(c). It is indicated that the residual control input $u$ during the settling motion area is correctly estimated by the augmented observer. These experimental results support the effectiveness of the proposed method.

\section{Conclusion}

This paper has presented an approach to reconstruct smooth output based on the quantized measurement in the presence of system disturbances. By fitting the quantized measurements with polynomials in a moving horizon manner, a smooth signal is reconstructed via solving a convex optimization problem, where $\ell_{1}$-norm regularization is exploited to automatically determine the degree of the polynomial. The information on the quantization and the system models is taken into account in the optimization. Its performance has been illustrated through numerical simulation. Furthermore, the proposed method has been implemented in a real high-precision linear stage through DSP, and its effectiveness is verified though the experiments using the real positioning system.

\section{REFERENCES}

1. Devasia, S., Eleftheriou, E., and Reza M.S.O., "A Survey of Control Issues in Nanopositioning," IEEE Trans. Control Syst. Technol., Vol. 15, No. 5, pp. 802-823 (2007).

2. Tian, E., Yue, D., and Peng, C., "Quantized output feedback control for networked control systems," Information Sciences, Vol. 178, No. 12, pp. 27342749 (2008).

3. Goodwin, G. C., Silva E. I., and Quevedo, D. E., "Analysis and Design of Networked Control Systems using the Additive Noise Model Methodology," Asian Journal of Control, Vol. 12, No. 4, PP. 443-459 (2010).

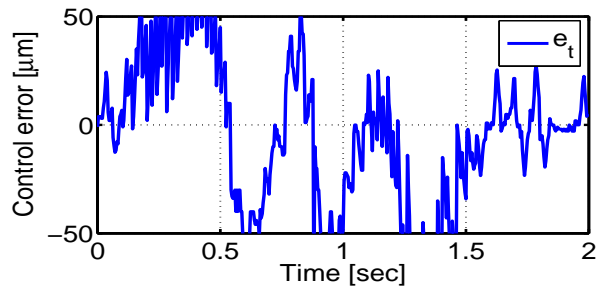

(a)

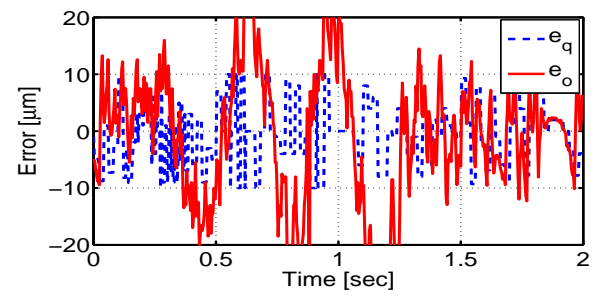

(b)

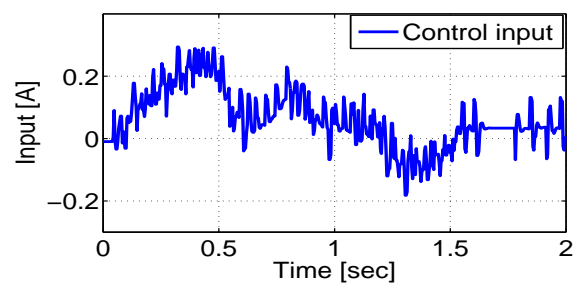

(c)

Fig. 15. Experimental results of Case II. (a) shows the position tracking error. (b) shows the estimation error. (c) shows the control input.

4. Jian, W., Zhibin, Y. and Jiafeng, Y. "Coding scheme based on boundary functions for distributed system with finite channel data rates and packet dropouts," Asian Journal of Control, doi: 10. 1002/.asjc.630 (2013).

5. Ling, Q., Gu, H., Lin, H., and Kang, Yu, "Bounds on the Optimal Quantization Performance of Dynamically Quantized Linear Systems with Bounded Noise," Asian Journal of Control, Vol. 14, No. 2, PP. 538-547 (2012).

6. Widrow, B., Kollar, I., and Liu, M., " Statistical Theory of Quantization," IEEE Trans. Instrum. Meas., Vol. 45, No. 2, pp. 353-361 (1996).

7. Brockett, R.W., and Liberzon, D., "Quantized feedback stabilization of linear systems," IEEE 


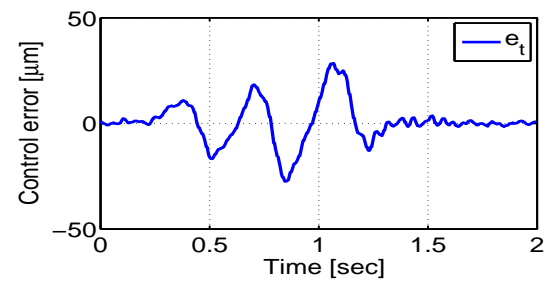

(a)

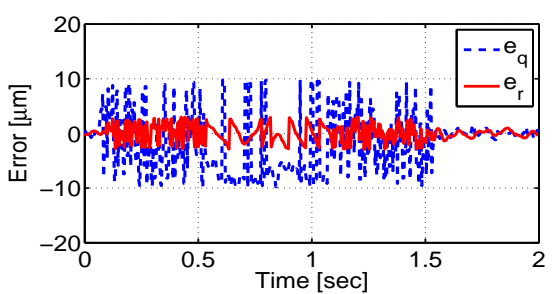

(b)

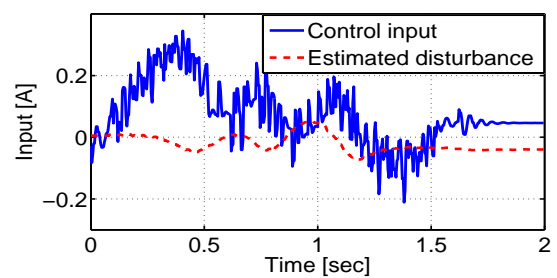

(c)

Fig. 16. Experimental results of Case III. (a) shows the position tracking error. (b) shows the quantization error and the reconstruction error, and (c) shows the control input and the estimated disturbance.

Trans. Autom. Control, Vol. 45, No. 7, pp. 12791289 (2000).

8. Fu, M., and Xie, L., "Finite-level quantized feedback control for linear systems," IEEE Trans. Autom. Control, Vol. 54, No. 5, pp. 1165-1170 (2009).

9. Xia, Y., Yan, J., Shang, Fu, M., and Liu, B., "Stabilization of quantized systems based on Kalman filter," Control Engineering practice, Vol. 20, No. 10, pp. 954-962 (2012).

10. Azuma, S. and Sugie, T., "ptimal Dynamic Quantizers for Discrete-Valued Input Control," Automatica, Vol. 44, No. 2, pp. 396-406 (2008).
11. Luong-Van, D., Tordon, M., and Katupitiya, J., "Covariance Profiling for an Adaptive Kalman filter to Suppress Sensor Quantization Effects," Proc. 43rd IEEE Conf. on Decis. Control, pp. 2680-2685 (2004).

12. Zhang, J., and Fu, M., "A Reset State Estimator for Linear Systems to Suppress Sensor Quantization Effects," Proc. 17th IFAC World Congress, pp. 9254-9259 (2008).

13. Hirata, M., and Kidokoro, T., "Servo Performance Enhancement of Motion System via a Quantization Error Estimation Method-Introduction to Nanoscale Servo Control," IEEE Trans. Ind. Electron., Vol. 56, No. 10, pp. 3817-3824 (2009).

14. Laakso, T.I., Tarczynski, A., Murphy, N.P., and Valimaki, V., "Polynomial Filtering Approach to Reconstruction and Noise Reduction of Nonuniformly Sampled Signals," Signal Processing, Vol. 80, No. 4, pp. 567-575 (2000).

15. Merry, R.J.E., van de Molengraft, M.J.G., and Steinbuch, M., "Velocity and acceleration estimation for optical incremental encoders," Mechatronics, Vol. 20, No. 1, pp. 20-26 (2010).

16. Zhu, H., and Sugie, T., "Velocity Estimation of Motion Systems Based on Low-Resolution Encoders," Trans. of the ASME, Journal of Dynamic Systems, Measurement and Control, Vol. 135, No. 1, pp. 011006-1-011006-8 (2013).

17. S. Boyd, L. Vandenberghe. Convex Optimization, Cambridge University Press, 2004.

18. Gautschi, W., "Optimally Conditioned Vandermonde Matrices," Numer. Math., Vol. 24, No. 1, pp. 1-12 (1975).

19. Che, W.-W., and Yang, G.-H., " $\mathrm{H}_{\infty}$ filter design for continuous-time systems with quantised signals," International Journal of Systems Science, DOI: 10.1080/00207721.2011.600473 (2004).

20. Fujimoto, H., Hori, Y., and Kawamura, A., "Perfect tracking control based on multirate feed forward control with generalized sampling periods," IEEE Trans. Ind. Electron., Vol. 48, No. 3, pp. 636-644 (2001).

21. Mattingley, J., Wang, Y., and Boyd, S., "Code Generation for moving horizon Control," Proc. IEEE Multi-Conf. Syst. Control, Japan, pp. 985992 (2010). 